\title{
Projet ReMed: rapport sur la phase pilote
}

\section{Comité de direction ReMed*}

* Michael Peltenburg, responsable du projet ReMed; Martina Hersperger, département DDQ FMH; Iris Stucki, département DDQ FMH; Werner Tschan, médecin spécialiste en psychiatrie et psychothérapie FMH.
Correspondance:

Dr Michael Peltenburg

Médecin spécialiste

en médecine générale FMH

Praxis Post

CH-8340 Hinwil

michael.peltenburg@hin.ch

\begin{abstract}
En bref
Les médecins exercent une profession à responsabilités. Leurs conditions de travail sont telles qu'elles peuvent conduire à de graves problèmes de santé psychique et physique. ReMed est une offre supplémentaire établie à l'intention de médecins confrontés à un syndrome d'épuisement et non habitués à demander de l'aide.

Avec ReMed, les médecins en situation de stress bénéficient d'une assistance professionnelle en temps utile, bien entendu sous garantie du secret médical. Une personne de confiance les conseille et établit, si nécessaire, des contacts avec des spécialistes aptes à leur procurer une assistance (mentorat ou coaching).

Le maintien des capacités fonctionnelles du médecin est au cœur du projet ReMed, qui a pour but d'inciter les médecins à prendre conscience, de manière accrue, des risques inhérents à leur profession. Conformément à leur engagement professionnel, ils sont tenus de prendre des mesures leur permettant d'assumer pleinement leurs responsabilités de médecin.

Le projet ReMed est opérationnel depuis octobre 2007 et quelques médecins en ont déjà fait usage.
\end{abstract}

\section{Réseau de soutien ReMed}

Les médecins exercent une activité à responsabilités. Ils travaillent de toutes leurs forces au maintien et au rétablissement de la santé de leurs patients. Leurs conditions de travail les exposent toutefois plus que d'autres à des facteurs de risques susceptibles de conduire à de graves problèmes de santé psychiques et physiques [1]. Les principaux facteurs de risque de nature institutionnelle sont les suivants: exigences professionnelles élevées, horaires longs et irréguliers, stress (manque de temps ou travail sous la pression du temps), nombreuses tâches administratives et attentes de plus en plus élevées des patients [2, 3]. C'est pourquoi de nombreux médecins s'occupent activement de leur santé, tiennent compte de leurs limites face au stress, veillent à mener une vie équilibrée entre travail et loisirs et recourent, le cas échéant, aux possibilités offertes en matière de supervision. La FMH prend ses responsabilités et soutient le corps médical en mettant à sa disposition une offre supplémentaire de soutien dans la gestion des situations difficiles. Ce nouveau réseau de soutien pour médecins s'appelle ReMed.

\section{Phase pilote}

Au printemps 2007, le Comité central de la FMH s'est prononcé en faveur d'un soutien financier au projet pilote ReMed (d'avril 2007 à septembre 2009), dont la mise en œuvre s'effectue, au cours d'une première phase, dans les cantons pilotes de Thurgovie et de Neuchâtel. ReMed a pour objectif de contribuer à la bonne santé des médecins en vue de garantir des soins médicaux de qualité élevée. Le réseau de soutien ReMed accompagne et soutient les médecins en situation de stress en leur offrant une assistance en temps utile. Les médecins qui cherchent de l'aide peuvent s'adresser à la personne de contact ReMed ou à une personne de confiance. Ces personnes sont des médecins de la région avec lesquels ils font, au cours d'un premier entretien, le constat de leur situation et définissent la démarche à adopter. Si nécessaire, la personne de confiance indique des collègues aptes à offrir un soutien approprié (mentorat ou coaching).

\section{Exemple}

Un médecin appelle pour dire qu'il est surchargé, que les travaux administratifs l'oppressent, qu'il n'a véritablement de temps ni pour ses patients ni pour sa famille.

La personne de contact ReMed fixe un rendezvous, examine la situation, détecte clairement les signes d'un épuisement professionnel et propose un mentorat. Après avoir hésité, le médecin concerné accepte cette offre puis communique, lorsqu'on le contacte pour lui poser la question, que les tensions ont déjà largement diminué à la maison.

Les offres sont adaptées aux besoins individuels et les médecins concernés ont le libre choix du spécialiste et du sexe de leur interlocuteur. Chez ReMed, les médecins à la recherche de conseils et d'assistance sont traités en tant que patients et 
bénéficient des mêmes droits. Toutes les mesures sont prises dans le respect des dispositions régissant la protection des données et le secret médical (art. 321 du Code pénal).

\section{Responsabilité professionnelle}

La philosophie de ReMed repose sur la responsabilité personnelle du médecin. Elle implique que les médecins soient attentifs aux facteurs de risque présents dans leur environnement professionnel et prennent connaissance des possibilités offertes par la promotion de la santé. Ce faisant, ils sont amenés à prendre des mesures leur permettant d'assumer pleinement leurs responsabilités professionnelles. Cette philosophie s'inspire de la Charte internationale du professionnalisme médical (Physicians' Charter [4]). Cette charte engageant la responsabilité du médecin a été mise au point en commun par les organisations médicales suivantes: European Federation of Internal Medicine, American College of Physicians, American Society of Internal Medicine et American Board of Internal Medicine. Sous le titre «Un ensemble de responsabilités professionnelles», les médecins prennent l'engagement suivant:

Commitment to professional responsibilities

As members of a profession, physicians are expected to work collaboratively to maximise patients' care, be respectful of one another, and participate in the process of self-regulation, including remediation and discipline of members who have failed to meet professional standards. The profession should also define and organize the educational and standardsetting process for current and future members. Physicians have both individual and collective obligations to participate in these processes. These obligations include engaging in internal assessment and accepting external scrutiny of all aspects of their professional performance.

\section{Figure 1}

Capacité fonctionnelle du médecin.

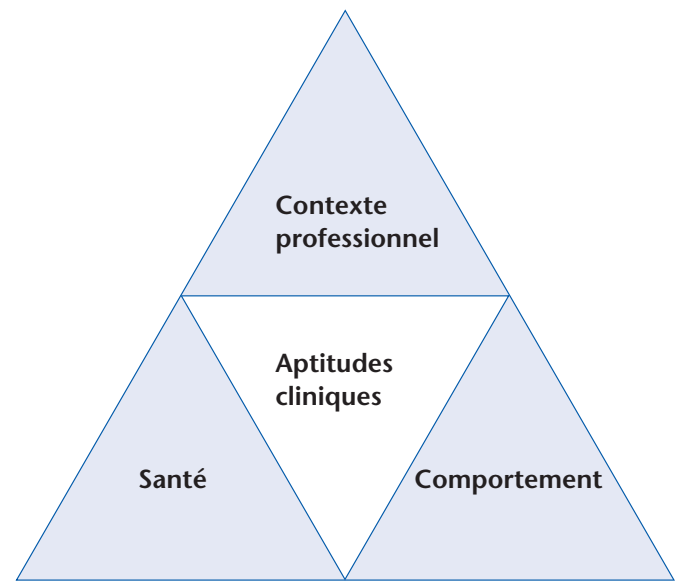

Source: adaptation d'après McAvoy [7].
De nombreux médecins éprouvent de la difficulté à endosser le rôle du patient et donc à changer de rôle (Rabin et al. 2005). Ils ne sont pas habitués à demander de l'aide, ils tentent de poser un diagnostic et de se soigner eux-mêmes lorsqu'ils sont confrontés à des problèmes professionnels et personnels (Fowlie 2005). Mais la crainte de la stigmatisation disparaît dès que les problèmes sont abordés de manière objective. Une vigilance collégiale réciproque et le courage de s'adresser aux personnes concernées sont d'autres aspects positifs de la discussion entre professionnels.

\section{Maintenir la capacité fonctionnelle}

Le terme de «capacité fonctionnelle» englobe tous les domaines que le médecin doit maîtriser dans l'exercice de sa profession (fig. 1):

- contexte professionnel: conditions de travail, équipe, conditions-cadres de l'organisation;

- santé: promotion de la santé et prévention;

- comportement: caractéristiques personnelles, aptitude à diriger et à résoudre des conflits, présentation personnelle;

- aptitudes cliniques: connaissances et compétences professionnelles, comportement.

ReMed tient à contribuer au maintien de la capacité fonctionnelle des médecins mais n'assume aucune fonction de contrôle. Lorsque des tiers doutent, à bon droit, de la capacité d'un médecin à exercer sa profession, ils peuvent s'adresser à ReMed qui prendra alors contact avec le praticien concerné. Néanmoins, toute intervention sous la forme d'une évaluation ne sera effectuée que sur mandat d'un organisme officiel (p.ex. une autorité cantonale), conformément aux exemples suivants:

\section{Exemple 1}

Dans un cabinet de groupe, une femme-médecin effectuant un remplacement apprend d'une patiente qu'un médecin du cabinet de groupe a fait preuve d'inconduite sexuelle à son égard. Lors d'une discussion interne, les médecins du groupe en parlent avec le médecin concerné qui nie tout et présente ladite patiente comme une menteuse notoire. Que peut faire ReMed?

ReMed propose un entretien au médecin concerné et tente de le convaincre de conclure un contrat et de se faire accompagner par un coach. S'il refuse, les médecins du cabinet ou la patiente doivent engager eux-mêmes d'autres démarches et s'adresser, p.ex., à un organisme officiel tel que le médecin cantonal. 


\section{Exemple 2}

Le fils d'une femme-médecin s'adresse à ReMed parce que sa mère, qui vit seule, s'adonne à la boisson.

Lors du premier entretien, ReMed accepte d'offrir son aide et invite le fils à prendre contact avec sa mère pour lui parler ouvertement de sa démarche, de ses soucis et de la disponibilité de ReMed à prendre contact avec elle. Le fils communique ensuite à ReMed que la discussion a permis de détendre clairement la situation et que sa mère a elle-même demandé de l'aide à un psychiatre.

\section{Exemple 3}

Le médecin cantonal s'adresse en dernier recours à ReMed en vue d'une évaluation chez un confrère alcoolique.

Le médecin concerné accepte de collaborer avec ReMed (dans le cas contraire, ReMed renvoie le cas au médecin cantonal avec toutes les conséquences qui en découlent pour le médecin concerné.) ReMed met sur pied une équipe multidisciplinaire chargée d'évaluer ce médecin quant à ses conditions de travail, sa santé, son comportement et ses capacités cliniques; elle lui propose ensuite des objectifs réalistes et choisit un coach avec lui. Le succès du contrat passé de manière partenariale sera examiné de manière externe à l'échéance fixée. Le médecin cantonal sera informé du succès ou de l'échec des mesures prises.

\section{Projet pilote opérationnel depuis octobre 2007}

Le projet pilote est entré dans sa phase opérationnelle à fin octobre 2007. Le comité de direction assume la direction stratégique et opérationnelle, tandis que la direction ReMed cantonale offre des prestations ReMed dans la région. Le site internet www.swiss-remed.ch renseigne sur l'offre ReMed, donne de brèves informations sur les problèmes professionnels et personnels auxquels les médecins peuvent être confrontés et met à disposition un formulaire permettant d'envoyer des demandes à ReMed. La ligne d'assistance téléphonique 0800073633 (ou 08000 ReMed, soit 08000 + le numéro correspondant aux lettres ReMed figurant sur l'appareil de téléphone), en service 24 heures sur 24 , prend note des coordonnées et demandes reçues et les transmet à la personne de confiance ReMed. Les demandes peuvent aussi être envoyées par courriel à info@swiss-remed.ch. Quelques médecins ont déjà fait usage du projet ReMed depuis son lancement.

\section{Références}

1 Keller R, Peltenburg M, Hersperger M, Hornung R. Résultats de l'étude de faisabilité concernant un réseau d'assistance pour les médecins. Bull Méd Suisses. 2007;88(21):867-73.

2 Bergner TMH. Burnout bei Ärzten. Stuttgart: Schattauer; 2006.

3 Goehring C, Bouvier Gallacchi M, Künzi B, Bovier P. Psychosocial and professional characteristics of burnout in Swiss primary care practitioners: a crosssectional survey. Swiss Med Wkly. 2005;135(7/8): 101-8.

4 Medical Professionalism Project. Medical professionalism in the new millennium: a physicians' charter. Lancet. 2002;359:520-2.

5 Rabin S, Matalon A, Maoz B, Shiber A. Keeping doctors healthy: a salutogenic perspective. Families Systems Health. 2005;23:94-102.

6 Fowlie DG. Doctors' drinking and fitness to practise. Alcohol Alcoholism. 2005;40:483-4.

7 McAvoy P. The NCAS Assessment Process. Exposé présenté dans le cadre de la session «Handling concerns about the performance of doctors» (31.1.-1.2.2008), Oslo. www.legeforeningen. no/index.gan? id $=134362 \&$ su bid=0 (accès 15 février 2008). 